\title{
EL EFECTO POTENCIAL DE LAS REMESAS EN LA POBREZA Y LA DESIGUALDAD
}

\author{
José Jorge Mora Rivera* \\ Alejandro López Feldman**
}

(Recibido: Agosto, 2010 / Aprobado: Octubre, 2010)

\begin{abstract}
RESUMEN
Una de las consecuencias directas de la crisis económica reciente de los Estados Unidos fue una disminución en el envío de remesas provenientes de ese país hacia México y en consecuencia algunos impactos sobre sus niveles de pobreza y desigualdad. Utilizando datos de la Encuesta Nacional a Hogares de México y técnicas de descomposición de indicadores de pobreza y desigualdad, este documento explora los impactos potenciales de una disminución en las remesas sobre la pobreza y la desigualdad. Los resultados sugieren que las remesas provenientes de los Estados Unidos tienen impactos significativos sobre los niveles de pobreza mientras que no se encuentran efectos importantes sobre la distribución del ingreso.

Palabras clave: Migración, remesas, desigualdad, pobreza, México Clasificación JEL: O15, I32
\end{abstract}

* Profesor-Investigador, Departamento de Economía, ITESM-CCM. Correo electrónico: $<$ jjmora@itesm.mx>.

**Profesor-Investigador, División de Economía, CIDE. Correo electrónico: <alejandro.lopez@cide. edu>. 
José Jorge Mora Rivera / Alejandro López Feldman

\begin{abstract}
The recent US economic crisis had as one of its consequences for Mexico a decrease in the reception of US remittances and as a result some impacts on inequality and poverty levels. This paper utilizes data from the Mexico National Rural Household Survey, together with inequality and poverty decomposition techniques, to explore the potential impacts of remittances on rural inequality and poverty if this source of income decreases. The results suggest that remittances from US have significant impacts on poverty levels while have no important effects on income distribution.

Keywords: Migration, remittances, inequality, poverty, Mexico

JEL Classification: O15, I32
\end{abstract}

\title{
1. INTRODUCCIÓN
}

Durante los últimos veinte años México ha experimentado un fuerte incremento en la migración de mano de obra rural hacia los Estados Unidos. Entre 1990 y 2002 la proporción de la población rural de México que se encontraba trabajando en los Estados Unidos aumentó del 7\% al 14\% (Mora y Taylor, 2005). Por su parte los flujos de remesas a México desde los Estados Unidos pasaron de menos de $\$ 700$ millones de dólares (0.3\% del PIB) en el año1980 a más de \$6 500 millones de dólares (1.1\% del PIB) en el 2000, para luego alcanzar \$16 600 millones de dólares (2.5\% del PIB) en el 2004 (López-Córdova, 2005) y registrando su máximo histórico en 2007 con una cifra superior a los \$26000 millones de dólares.

Una proporción importante de hogares en todo el país se ven beneficiadas por estos flujos de ingresos (cerca del $4.4 \%$ de los hogares recibieron ingresos por remesas de los Estados Unidos en el 2000; ibid.). Los ingresos por remesas pueden representar una fracción importante de los ingresos de los hogares en México y, por lo tanto, pueden tener un impacto tanto en los niveles de pobreza como en la desigualdad del ingreso. 
La reciente crisis mundial que afectó fuertemente a la economía estadounidense generó un impacto negativo en el flujo de remesas recibidas en México. Según cifras del Banco de México, el flujo de remesas en 2009 respecto a 2007 cayó en \$4 870 millones de dólares y se espera que dicha tendencia continúe para el 2010.

El objetivo principal del presente documento es analizar los impactos de corto plazo que una disminución en el envío de remesas provenientes de Estados Unidos podría tener en los niveles de pobreza y desigualdad en el México rural. Para lograr lo anterior se recurre al análisis de medidas de pobreza y desigualdad utilizando los datos de la Encuesta Nacional a Hogares Rurales de México (ENHRUM).

Además de la presente introducción, el documento contiene cuatro secciones adicionales. En la segunda sección se presenta la metodología empleada en materia de indicadores de pobreza y desigualdad. En la tercera sección se describe la base de datos empleada; los resultados de las simulaciones implementadas se presentan en la cuarta sección y, en la última sección se ofrecen las conclusiones que surgen del análisis efectuado en el documento.

\section{METODOLOGÍA}

\section{a) Pobreza}

Para analizar el impacto que la disminución de remesas provenientes de los Estados Unidos podría tener en los niveles de pobreza en el México rural, se utilizan las tres variantes más comunes del índice de pobreza propuesto por Foster, Greer y Thorbecke en 1984 (FGT). La fórmula general del índice FGT es la siguiente:

$$
F G T(\alpha)=\frac{1}{N} \sum_{i=1}^{N} I_{i}\left(1-\frac{y_{i}}{z}\right)^{\alpha}
$$

donde $I_{i}=1$ si $y_{i} \leq z$ y es cero en cualquier otro caso, $z$ es la línea de pobreza, $n$ es el número total de hogares; $y_{i}$ es el ingreso equivalente del que dispone el hogar $i$ y $\alpha$ es un parámetro que refleja la importancia que se le da, en términos relativos, al ingreso de los más pobres. Cuando $\alpha=0$, la fórmula se colapsa en lo que se conoce como la incidencia de la pobreza (en este caso, es la proporción de 
hogares pobres respecto al total de hogares considerados), cuando $\alpha=1$ se obtiene la profundidad de la pobreza y cuando $\alpha=2$ se obtiene la severidad de la pobreza.

El índice FGT puede descomponerse de acuerdo a las distintas fuentes de ingreso que componen el ingreso total. Digamos que $Y_{d}=\left(Y_{d l}, Y_{d 2}, \ldots, Y_{d l}\right)$ representa el ingreso de los hogares en orden creciente y $z>0$ denota una línea de pobreza predeterminada. El índice FGT puede definirse como:

$$
P\left(Y_{d} ; z\right)=\frac{1}{n z^{\alpha}} \sum_{i=1}^{q} g_{i}^{\alpha}
$$

donde $n$ es el número de hogares, $q=q\left(Y_{d} ; z\right)$ es el número de hogares pobres, la brecha entre el ingreso del hogar y la línea de la pobreza está dada por $g_{i}=z-Y_{d i}$, y $\alpha$ es un parámetro. En este trabajo nos enfocaremos a los efectos potenciales en: la incidencia de la pobreza $(\alpha=0)$, la brecha de la pobreza $(\alpha=1)$ y la severidad de la pobreza $(\alpha=2)$.

Para descomponer $P\left(Y_{d} ; z\right)$ por fuentes de ingreso sustituimos $Y_{d}$ por la suma de ingresos de cada fuente $i$. Esto nos da como resultado:

$$
P\left(Y_{d} ; z\right)=\frac{1}{n z^{\alpha}} \sum_{i=1}^{q}\left(z-\sum_{k=1}^{K} y_{k}\right)^{\alpha}
$$

El impacto en el nivel de pobreza originado por un cambio porcentual en una fuente de ingreso se puede obtener mediante

$$
\frac{d P\left(Y_{d}, e ; z\right)}{d e}=\frac{1}{n z^{\alpha}}\left[\sum_{i=1}^{q_{0}}-\alpha g_{i}(e)-\sum_{q-} g_{i}(e)^{\alpha}+\sum_{q^{+}} g_{i}(e)^{\alpha}\right]
$$

Además de simular los cambios en los niveles de pobreza que podrían observarse como consecuencia de cambios porcentuales en la percepción de remesas, se realizó un análisis econométrico para obtener más información sobre la relación existente entre la recepción de remesas internacionales y la probabilidad de que un hogar sea pobre así como la relación entre pobreza y algunas de las características básicas del hogar. Para lograr esto se recurrió a un modelo Probit en el cual la variable dependiente es una variable dicotómica que toma el valor de uno si el hogar es pobre y de cero si no lo es. 


\section{b) Desigualdad}

Para el análisis del efecto que un cambio en los niveles de remesas podría tener en la desigualdad se recurre al coeficiente de Gini y, en particular, a la descomposición del coeficiente de Gini por fuentes de ingreso, sugerida por Lerman y Yitzhaki (1985).

Si consideramos que $y_{1}, \ldots, y_{k}$ son los $k$ componentes del ingreso del hogar; si $Y$ representa el ingreso total del hogar, entonces

$$
Y=\sum_{k=1}^{K} y_{k}
$$

Siguiendo a Pyatt, et al. (1980) y a Lerman y Yitzhaki (1985), se puede obtener el coeficiente de Gini como una función de la covarianza entre el ingreso y su función de distribución acumulativa, esto es:

$$
G=\frac{2 \operatorname{Cov}[Y, F(Y)]}{\mu_{Y}}
$$

$G$ es el coeficiente de Gini del ingreso total, $\mu_{Y}$ representa el ingreso promedio y $F(Y)$ es la función de distribución acumulativa del ingreso total. La ecuación (5) se puede escribir como:

$$
G=\frac{2 \sum_{k=1}^{K} \operatorname{Cov}\left[y_{k}, F(Y)\right]}{\mu_{Y}}=\sum_{k=1}^{K} S_{k} G_{k} R_{k}
$$

donde $S_{k}$ representa la proporción del ingreso $k$ en el ingreso total, $G_{k}$ es el índice de Gini correspondiente a la fuente $k$ y $R_{k}$ es la correlación entre el ingreso de la fuente $k$ con el ingreso total.

La ecuación (6) permite descomponer el papel que cualquier fuente de ingreso (en este caso las remesas) tiene sobre la desigualdad en un conjunto de tres términos fáciles de interpretar:

i) la magnitud de la fuente de ingreso en relación al ingreso total;

ii) la desigualdad misma de la fuente de ingreso;

iii) la correlación de la fuente de ingreso con el ingreso total. 
Si se toman las decisiones de producción y trabajo de los hogares como dadas y se considera un cambio exógeno en el componente $j$-ésimo del ingreso del hogar por un factor $\pi$, tal que $y_{j}(\pi)=(1+\pi) y_{j}$, entonces

$$
\frac{\partial G}{\partial \pi}=S_{j}\left(R_{j} G_{j}-G\right)
$$

donde $S_{j}, G_{j}, G$ y $R_{j}$ denotan la participación del ingreso j-ésimo en el ingreso total, los coeficientes de Gini y la correlación de Gini antes del cambio en el ingreso marginal.

Si se divide por el coeficiente de Gini inicial, $G$, se tiene:

$$
\frac{\partial G / \partial \pi}{G}=\frac{S_{j} R_{j} G_{j}}{G}-S_{j}
$$

La ecuación (8) indica que el efecto relativo de un cambio porcentual marginal en el componente j-ésimo sobre la desigualdad es igual a la contribución relativa del componente $j$, a toda la desigualdad, menos la contribución relativa al ingreso total. Es fácil ver que mientras las remesas sean un componente importante en el ingreso de los hogares, entonces:

- Si la correlación de Gini entre las remesas y el ingreso total, $R_{j}$, es negativa o cero, un incremento en las remesas necesariamente disminuye la desigualdad.

- Si la correlación de Gini es positiva, entonces el impacto sobre la desigualdad depende del signo de $R_{j} G_{j}$ - $G$. Una condición necesaria para que la desigualdad aumente es que la desigualdad de las remesas debe exceder la desigualdad del ingreso total del hogar, es decir $G_{j}>G$, puesto que $R_{j} \leq 1$.

Utilizando esta metodología es posible estimar el efecto que cambios porcentuales en las remesas tendrían en la desigualdad del ingreso de los hogares rurales mexi- 
canos, dejando los ingresos de todas las demás fuentes constantes (Stark, Taylor y Yitzhaki, 1986).

\section{DATOS}

Para realizar la investigación se utilizarán datos de la Encuesta Nacional a Hogares Rurales de México (ENHRUM). La encuesta se aplicó entre enero y febrero del 2003 y fue coordinada por miembros del Programa de Estudios del Cambio Económico y de la Sustentabilidad del Agro Mexicano (PRECESAM) de El Colegio de México (COLMEX) y del Rural Economies of the Americas Program (REAP) de la Universidad de California en Davis (UCD). En su levantamiento participaron más de 100 investigadores jóvenes y estudiantes de 11 universidades e instituciones académicas de los estados del país.

El objetivo de la ENHRUM fue obtener por vez primera información representativa en el plano nacional sobre la economía y sociedad rurales de México y, con ella, elaborar estudios empíricos sobre los efectos de las reformas agropecuarias y comerciales en la producción, ingreso y migración de los hogares y del sector rural. Esta muestra provee información detallada sobre: activos, características socio-demográficas, producción, fuentes de ingresos y migración, de una muestra nacional representativa de hogares rurales.

El diseño muestral de la encuesta fue realizado por el Instituto Nacional de Estadística, Geografía e Informática (INEGI). La cobertura geográfica de la ENHRUM es a nivel nacional en poblaciones rurales de 500 a 2499 habitantes; por razones de factibilidad y de costo, individuos de poblaciones dispersas menores a 500 habitantes no fueron incluidos en la muestra. El resultado es una muestra

representativa de más del $80 \%$ de la población, que el censo oficial de México considera como rural. Para implementar la muestra, México fue dividido en 5 regiones reflejando la regionalización estándar del país: centro, sur-sureste, centrooccidente, noroeste y noreste. La encuesta se aplicó en 80 comunidades de 14 estados del país. La Tabla 1 muestra la distribución de las comunidades que fueron encuestadas. 
José Jorge Mora Rivera / Alejandro López Feldman

TABLA 1

Distribución de las comunidades encuestadas en la muestra

\begin{tabular}{|c|c|c|}
\hline Regiones & Estados & Localidades \\
\hline Sur-sureste & $\begin{array}{l}\text { Oaxaca, } \\
\text { Veracruz } \\
\text { Yucatán } \\
\end{array}$ & 16 \\
\hline Centro & $\begin{array}{c}\text { Estado de México } \\
\text { Puebla }\end{array}$ & 16 \\
\hline Centro-occidente & $\begin{array}{c}\text { Guanajuato } \\
\text { Nayarit } \\
\text { Zacatecas }\end{array}$ & 16 \\
\hline Noroeste & $\begin{array}{c}\text { Baja California } \\
\text { Sonora } \\
\text { Sinaloa }\end{array}$ & 16 \\
\hline Noreste & $\begin{array}{c}\text { Chihuaha } \\
\text { Durango } \\
\text { Tamaulipas }\end{array}$ & 16 \\
\hline TOTAL & 14 & 80 \\
\hline
\end{tabular}

Fuente: Elaboración propia.

En el presente trabajo se utiliza información para 1745 hogares rurales. Al tomar en cuenta los factores de expansión, que reflejan el número de hogares que cada hogar entrevistado representa, se tiene que la muestra representa los ingresos recibidos durante el 2002 por 2987994 hogares.

En general, la ENHRUM sigue las definiciones de la ENIGH 2002 de la mayor parte de los conceptos de las variables sociodemográficas y económicas; por ejemplo: edad, sexo, nivel de instrucción, relación de parentesco, remuneración al trabajo, autoconsumo, ingreso, gastos, etc. Sin embargo, a partir de las características particulares de los hogares rurales y de los objetivos propios de la encuesta, se definen para la ENRHUM un mayor número de conceptos e información más precisa de las distintas actividades generadoras de ingresos y gastos en las que los hogares rurales mexicanos participan. Por ejemplo, es posible obtener información más detallada del fenómeno migratorio y de los flujos monetarios que éste 
origina. En la ENIGH 2002 solamente es posible conocer las transferencias que los hogares reciben ya sean provenientes del exterior o del interior del país, siendo esta la mejor aproximación que se tiene en relación a los montos de remesas que los hogares captan, en cambio la ENHRUM contiene información directa de las remesas recibidas en el hogar por cada uno de los miembros que forman parte del mismo. Ello posibilita tener información de mayor calidad al utilizar datos de la ENHRUM en relación al ENIGH, especialmente para lograr los objetivos que la presente investigación persigue.

Los datos obtenidos permiten calcular el ingreso total para cada uno de los hogares de la muestra. El ingreso total se definió como la suma de cinco fuentes de ingreso: producción familiar (agricultura, ganadería, extracción de recursos naturales y servicios); trabajo asalariado; remesas internas; remesas de los Estados Unidos y; transferencias del gobierno.

El ingreso neto de las actividades productivas de los hogares, con excepción del ingreso por ganado, fue estimado como el valor bruto de la producción, menos los insumos comprados. La producción no sólo incluye la producción comercial, también la producción consumida en el hogar y dada a otras familias como regalos.

Con el fin de obtener el valor bruto de la producción comercial, a los hogares se les preguntó el precio al que ellos vendieron sus productos. Para la producción consumida en casa o dada a otras como regalo, a los hogares se les preguntó el precio que ellos recibirían por vender el producto. La leña y otros bienes producidos para el consumo en el hogar fueron valorados preguntándoles a los hogares qué precio deberían pagar para comprar estos bienes.

El ingreso por la producción de ganado fue estimado como el cambio en el valor del ganado en pie entre el final y el comienzo del año en que se realizó la encuesta, mas (a) ventas y regalos a otros hogares de animales y productos de animales y (b) el consumo del hogar de animales criados en éste y productos animales; menos (c) compras de ganado y, (d) el costo de los insumos para el ganado (alimento, medicinas, y otros costos). Los salarios fueron agregados a través de los miembros del hogar y de los trabajos desempeñados por cada miembro.

No es clara la forma de valorar el insumo de las familias, como el trabajo, los animales y el equipo usado en la producción de actividades específicas. Debido 
a esto, no se intentó imputar el valor de los insumos familiares. Por otro lado, se permitió la posibilidad de que los hogares tuvieran ingresos netos con valor de cero o con valores negativos en actividades específicas.

En todas las estimaciones que se presentan en este trabajo los ingresos netos que se utilizan son ingreso por adulto equivalente. Para obtener dichas equivalencias se utilizó la escala de equivalencia de Rothbarth siguiendo lo sugerido por Teruel, Rubalcava y Santana (2005).

Para el cálculo de los índices de pobreza se recurrió a los datos del CONEVAL (2006). En específico se utilizó la línea de pobreza rural alimentaria que en precios de agosto del 2002 es de 494.78 pesos mensuales.

La Tabla 2 muestra los valores promedio de los ingresos (totales y por fuente) de los hogares rurales mexicanos que reciben remesas de los Estados Unidos y los

\section{TABLA 2}

\section{Ingresos promedio totales y por fuentes}

\begin{tabular}{l|c|c|}
\hline & $\begin{array}{c}\text { Hogares que no reciben } \\
\text { remesas de Estados Unidos }\end{array}$ & $\begin{array}{c}\text { Hogares que reciben } \\
\text { Remesas de Estados Unidos }\end{array}$ \\
\hline $\begin{array}{l}\text { Ingreso total } \\
\text { Ingreso por producción }\end{array}$ & 11,969 & $18,529 * * *$ \\
familiar & 3,873 & 5,202 \\
Ingreso por trabajo & 6,525 & $4,167 * * *$ \\
asalariado & 1,101 & $1,802 * * *$ \\
Ingreso por transferencias & 471 & 362 \\
$\begin{array}{l}\text { Remesas internas } \\
\text { Remesas de }\end{array}$ & 0 & $6,997 * * *$ \\
$\begin{array}{l}\text { Estados Unidos } \\
\text { Porcentaje de hogares } \\
\text { por debajo de la } \\
\text { línea de pobreza }\end{array}$ & $44.5 \%$ & $19.6 \%$ \\
\hline $\mathrm{N}$ (hogares entrevistados) & $2,519,972(1,442)$ & $468,022(303)$ \\
\hline
\end{tabular}

*** La diferencia entre medias es estadísticamente significativa al 1\% utilizando errores estándar robustos a nivel de cluster.

Los ingresos presentados son ingresos anuales equivalentes en pesos de agosto del 2002.

Fuente: Elaboración propia. 
ingresos de los hogares que no lo hacen. El ingreso total de los hogares que reciben remesas de los Estados Unidos es superior en más del 50\% que el ingreso de los hogares que no las reciben. Los hogares que reciben remesas también reciben más transferencias del gobierno (alrededor de 60\% más). Por otro lado, los ingresos por salarios son casi $60 \%$ más altos en los hogares que no reciben remesas. Las diferencias en ingreso por producción familiar y en remesas internas no son estadísticamente significativas.

\section{RESULTADOS}

La Tabla 2 nos muestra que el $44 \%$ de los hogares rurales que no reciben remesas de los Estados Unidos son pobres, mientras que únicamente el $20 \%$ de los que reciben remesas están por debajo de la línea de pobreza alimentaria. Esto nos da una primera aproximación del impacto potencial que los cambios en las remesas provenientes de los Estados Unidos podrían generar en el ingreso de los hogares rurales de México.

En este sentido el escenario más dramático que se puede plantear es uno en el que los hogares dejan de recibir por completo las remesas de los Estados Unidos. Los efectos que esto habría tenido en los niveles de ingreso de los hogares rurales de México en el año 2002, y por lo tanto en los niveles de pobreza y desigualdad, se capturan en la Figura 1.

Sin las remesas de los Estados Unidos la incidencia de la pobreza habría sido $10 \%$ mayor a lo que fue, esto quiere decir que sin las remesas de los Estados Unidos el número de hogares rurales por debajo de la línea de pobreza alimentaria habría aumentado en casi 125 mil hogares. El FGT(1) y el FGT(2) muestran también una alta sensibilidad a la pérdida de esta fuente de ingresos con incrementos del $15 \%$ y $13 \%$ respectivamente. Lo que nos indica que la pérdida total de remesas de los Estados Unidos no sólo haría que algunos hogares se volvieran pobres sino que haría que éstos se volvieran aún más vulnerables. La desigualdad en la distribución del ingreso en las comunidades rurales del país aumentaría en un 3\%. Todos estos cambios son estadísticamente significativos. 


\section{Figura 1}

\section{Cambio en los índices de pobreza y desigualdad si se excluyen las remesas provenientes de Estados Unidos}

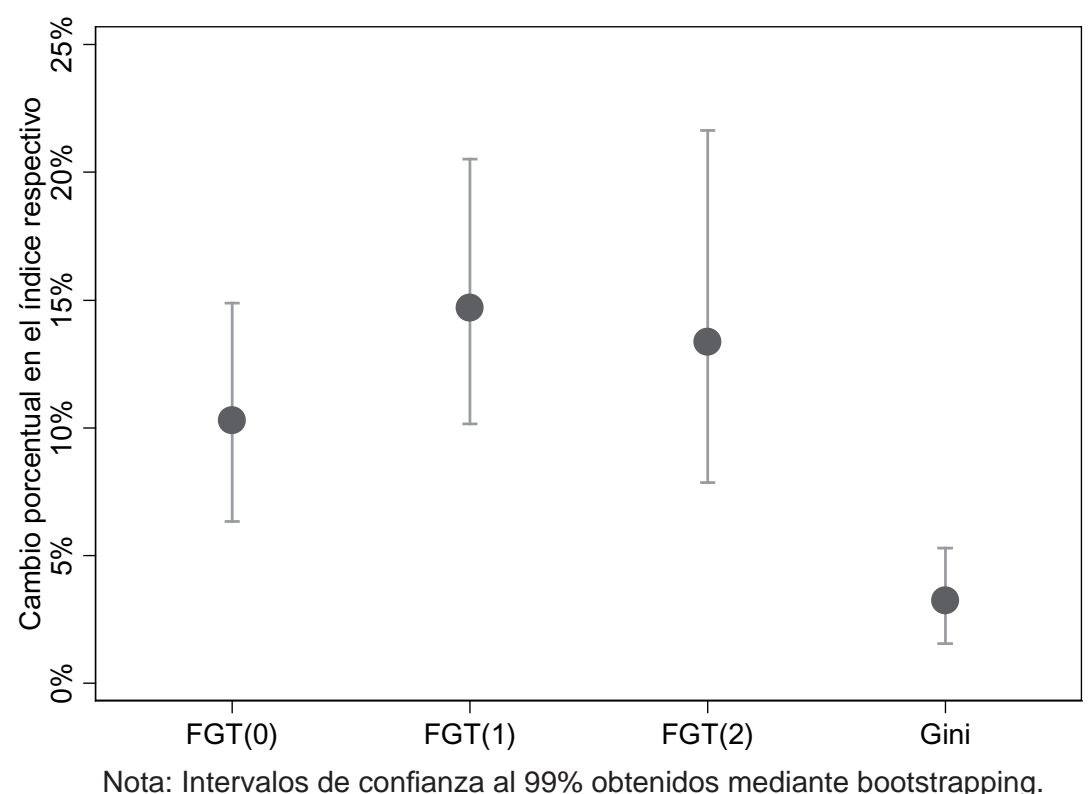

Es importante enfatizar que las disminuciones en remesas a las que nos referiremos afectan únicamente el ingreso de $16 \%$ de los hogares representados en nuestra muestra, es decir, únicamente a los hogares que recibieron remesas de los Estados Unidos en el 2002. Esto quiere decir que casi el 30\% de esos hogares quedarían por debajo de la línea de pobreza si perdieran dicha fuente de ingreso.

Por otro lado debemos mencionar que, el alcance del método de simulación utilizado en este trabajo, en el que se pierden las remesas internacionales (o una fracción de ellas) sin que cambien otras fuentes de ingresos, es limitado. Por un lado, podría argumentarse que en algunos casos una pérdida completa de remesas de los Estados Unidos se vería acompañada del retorno de al menos un migrante al 
hogar. Dicho migrante presumiblemente contribuiría a la generación de ingresos en el hogar, por lo cual el cálculo que estamos haciendo sobreestimaría el efecto negativo en el ingreso (al menos en el mediano plazo). Por otro lado, es posible que las remesas de los Estados Unidos estén permitiendo financiar alguna actividad productiva del hogar, por lo cual, al perderse dicha fuente los ingresos por otras fuentes podrían también verse disminuidos. En este caso nuestro método resultaría en una subestimación del efecto negativo en el ingreso total.

El siguiente paso en este ejercicio consiste en variar la proporción de remesas que los hogares receptores perderían. Dadas las limitaciones antes mencionadas esto puede verse como un ejercicio relativamente menos limitado ya que se puede argumentar que, en promedio, estas disminuciones reflejan no una situación en la que el migrante regresa al hogar sino una en la que por la situación económica dentro de los Estados Unidos se ve forzado a enviar menos remesas a México.

La Tabla 3 muestra los efectos que los distintos cambios porcentuales en la cantidad de remesas enviadas tendrían en los índices de pobreza. Los resultados muestran que incluso una caída del 10\% en las remesas tendría un efecto moderado en los índices de pobreza. La Figura 2 muestra cómo se modifica la incidencia de la pobreza ante distintos cambios en las remesas de los Estados Unidos. En esta figura podemos ver que la incidencia de la pobreza, ante una disminución del 30\% de las remesas, sería del 41.6\%, lo que implica un incremento de casi 30 mil hogares respecto a la situación base; mientras que una disminución del 60\% 1levaría a un incremento de más de 62 mil hogares.

En lo que a desigualdad del ingreso se refiere, la Figura 1 nos muestra que el cambio porcentual en la misma es relativamente pequeño, aún en el escenario de pérdida total de remesas. En este caso se decidió realizar un análisis de descomposición de desigualdad por fuentes del ingreso para obtener el efecto que un cambio marginal, en distintas fuentes de ingreso, tendría en el coeficiente de Gini. Los resultados de este ejercicio se muestran en la Tabla 4.

En la Tabla 4 es posible observar que, la participación de las remesas de los Estados Unidos y las nacionales en el ingreso total de los hogares rurales mexicanos son de $8.4 \%$ y $3.5 \%$, respectivamente. La principal fuente de ingreso proviene de los salarios, seguida por las actividades familiares que cuentan con un $31.4 \%$ 


\section{TABLA 3}

Índices de pobreza bajo diversos escenarios de disminución porcentual en la recepción de remesas provenientes de Estados Unidos

\begin{tabular}{|c|c|c|c|}
\hline $\begin{array}{c}\text { Disminución } \\
\text { porcentual }\end{array}$ & FGT(0) & FGT(1) & FGT(2) \\
\hline $0 \%$ & 0.406 & 0.245 & 0.270 \\
\hline $10 \%$ & 0.409 & 0.246 & 0.271 \\
\hline $20 \%$ & 0.412 & 0.247 & 0.273 \\
\hline $30 \%$ & 0.416 & 0.249 & 0.274 \\
\hline $40 \%$ & 0.422 & 0.251 & 0.276 \\
\hline $50 \%$ & 0.426 & 0.254 & 0.278 \\
\hline $60 \%$ & 0.427 & 0.257 & 0.281 \\
\hline $70 \%$ & 0.433 & 0.261 & 0.284 \\
\hline $80 \%$ & 0.439 & 0.267 & 0.288 \\
\hline $90 \%$ & 0.443 & 0.272 & 0.295 \\
\hline $100 \%$ & 0.447 & 0.281 & 0.306 \\
\hline & & & \\
\hline
\end{tabular}

Fuente: Elaboración propia. 


\section{FIGURA 2}

\section{Cambio en la incidencia de la pobreza ante disminuciones de las remesas provenientes de Estados Unidos}

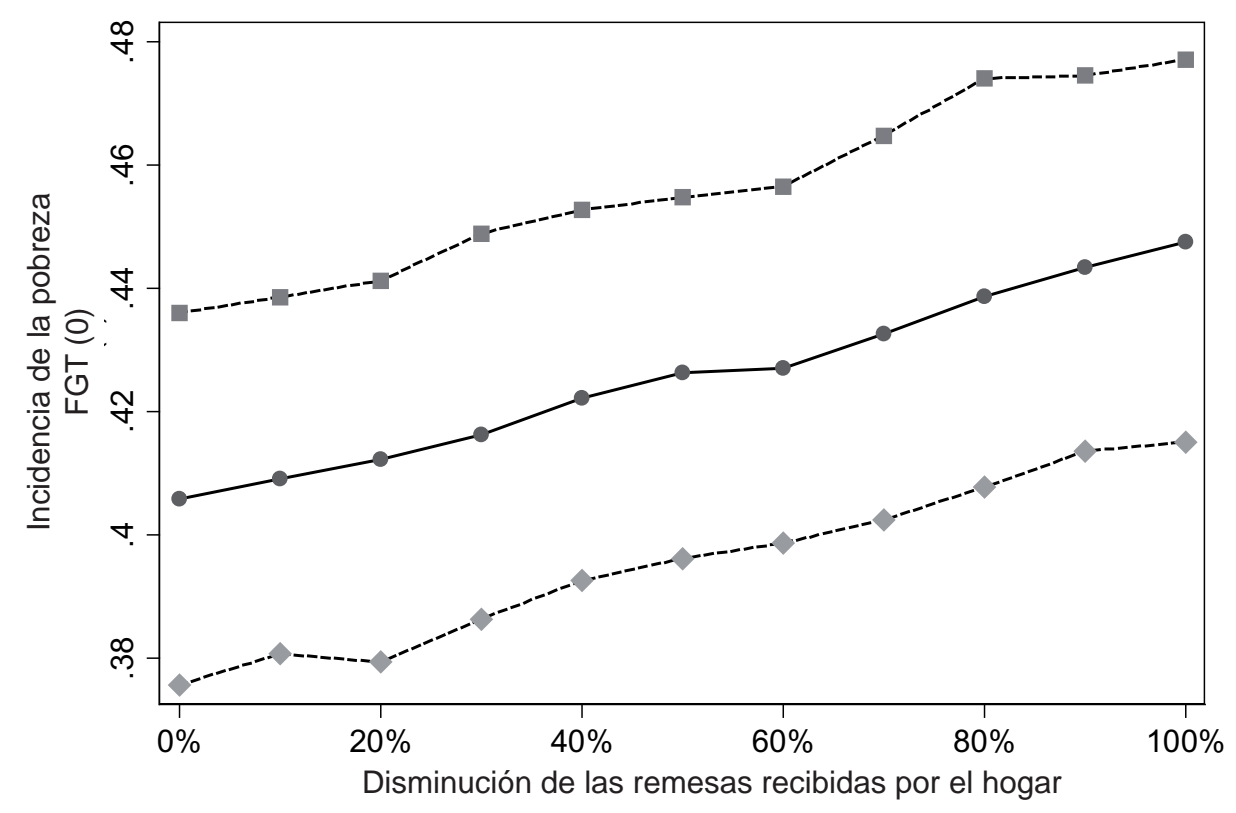

Nota: Las línes punteadas representan las bandas de confianza del 95\% (obtenidas mediante técnicas de bootstrapping).

del ingreso total. Las remesas nacionales muestran una mejor distribución que las remesas de los Estados Unidos (tercera columna de la Tabla 4) y su impacto en el coeficiente de Gini es negativo, señalando una mejora en la distribución del ingreso ante un cambio marginal en esta fuente. El resultado es contrapuesto para las remesas provenientes de los Estados Unidos, las cuales tienen un efecto positivo en el coeficiente de Gini, lo cual indica que la distribución empeora. Dichos resultados pueden ser explicados cuando analizamos la cuarta columna de la Tabla $4\left(R_{k}\right)$; en ella es posible observar que la correlación con el ingreso total es bas 
tante baja para las remesas del interior del país, lo que indica que son los hogares con menos ingresos los que están percibiendo esta fuente de ingresos y por ello su efecto al reducir la inequidad. Por su parte, las remesas de los Estados Unidos presentan una correlación mucho mayor (0.63) con el ingreso total, ello indica que los hogares que están recibiendo dicha fuente no son los de menores ingresos y, por lo tanto, el efecto resultante es de ampliar la brecha en la distribución del ingreso de los hogares rurales de México. En síntesis, los principales resultados sobre remesas y desigualdad indican que, para México las remesas internacionales incrementan la desigualdad del ingreso rural, mientras que el impacto de las remesas nacionales es equilibrador (última columna de la Tabla 4).

\section{TABLA 4}

\section{Descomposición de la desigualdad por fuentes de ingreso}

\begin{tabular}{|c|c|c|c|c|c|}
\hline Fuente & Sk & Gk & $\mathrm{Rk}$ & $\begin{array}{c}\text { Proporción } \\
\text { del gasto }\end{array}$ & $\%$ Cambio \\
\hline $\begin{array}{l}\text { Actividades } \\
\text { familiares }\end{array}$ & 0.3140 & 1.0314 & 0.7911 & 0.4407 & 0.1267 \\
\hline Salarios & 0.4736 & 0.6980 & 0.7303 & 0.4153 & -0.0583 \\
\hline Transferencias & 0.0932 & 0.7171 & 0.3655 & 0.0420 & -0.0512 \\
\hline $\begin{array}{l}\text { Remesas } \\
\text { Estados Unidos }\end{array}$ & 0.0843 & 0.9384 & 0.6381 & 0.0868 & 0.0025 \\
\hline $\begin{array}{l}\text { Remesas México } \\
\text { Ingreso Total }\end{array}$ & 0.0349 & $\begin{array}{l}0.9216 \\
0.5813\end{array}$ & 0.2735 & 0.0151 & -0.0198 \\
\hline
\end{tabular}

Fuente: Elaboración propia.

Por último, se realizó un análisis econométrico utilizando un modelo Probit para estimar la correlación entre algunas variables de interés, entre ellas una variable dicotómica que toma el valor de uno si el hogar fue receptor de remesas de los Estados Unidos en el 2002, y la probabilidad de que un hogar se encuentre por 
debajo de la línea de pobreza. Es necesario aclarar que esta es una estimación muy cruda en la cual no se busca obtener una relación de causalidad debido al evidente problema de endogeneidad entre la variable dicotómica y el status de pobreza del hogar, entre otras cosas.

La Tabla 5 nos muestra una clara relación negativa entre recibir remesas y la probabilidad de estar por debajo de la línea de pobreza; un hogar que recibe remesas es $18 \%$ menos propenso a ser catalogado como pobre que uno que no recibe. La escolaridad del jefe del hogar también tiene una relación negativa con la probabilidad de ser pobre. Los hogares cuyo jefe no habla español tienen una probabilidad mayor a ser pobres, lo mismo es cierto para hogares que están a cargo de una mujer. El número de menores de edad en el hogar también aumenta la probabilidad de que el hogar sea pobre mientras que el número de adultos la disminuye. En lo que respecta a las regiones se puede observar que, respecto a la región Noreste, los hogares en la región Sur-sureste son más propensos a ser pobres mientras que los hogares en el resto de las regiones presentan una probabilidad menor de estar por debajo de la línea de pobreza.

\section{CONCLUSIONES}

Las remesas de los Estados Unidos son claramente una fuente importante de ingreso para los hogares que las reciben y contribuyen a disminuir y prevenir la pobreza de ingresos. Los resultados presentados en este documento nos muestran una estimación de los efectos que, en el corto plazo, podría tener en la pobreza y la desigualdad en el México rural una disminución de las remesas provenientes de los Estados Unidos.

Por un lado, estos resultados pueden verse como el máximo impacto posible dado que es de esperarse que en el mediano plazo los hogares cambien sus estrategias productivas para hacer frente a la pérdida de remesas (ya sea parcial o total). Por el otro, es posible que las remesas tengan un impacto positivo en otras actividades productivas del hogar, por lo que el efecto neto de la pérdida de remesas podría ser incluso mayor al que aquí se calcula. 


\section{TABLA 5}

Probabilidad de que un hogar se encuentre por debajo de la línea de pobreza

\begin{tabular}{|c|c|c|c|c|}
\hline & & Probit & & \\
\hline & $\begin{array}{c}\text { Coeficiente } \\
\text { [Errores estándar] }\end{array}$ & & $\begin{array}{c}\text { Efectos Marginales } \\
\text { [Errores estándar] }\end{array}$ & \\
\hline Recibió remesas & -0.51 & $* * *$ & -0.18 & $* * *$ \\
\hline & {$[0.12]$} & & {$[0.04]$} & \\
\hline $\begin{array}{l}\text { Años escolaridad } \\
\text { del } \\
\text { jefe del hogar }\end{array}$ & -0.05 & $* * *$ & -0.02 & $* * *$ \\
\hline & {$[0.01]$} & & [0.005] & \\
\hline Género ( $1=$ hombre $)$ & $\begin{array}{r}-0.29 \\
{[0.12]}\end{array}$ & $* *$ & $\begin{array}{r}-0.11 \\
{[0.05]}\end{array}$ & $* *$ \\
\hline $\begin{array}{l}\text { El jefe habla } \\
\text { español }\end{array}$ & -0.34 & $*$ & -0.13 & $*$ \\
\hline & {$[0.17]$} & & {$[0.07]$} & \\
\hline Tierra (Ha) & $\begin{array}{r}-0.01 \\
{[0.01]}\end{array}$ & & $\begin{array}{r}-0.004 \\
{[0.002]}\end{array}$ & \\
\hline $\begin{array}{l}\text { Menores que } \\
\text { habitan en el hogar }\end{array}$ & 0.10 & $* * *$ & 0.04 & $* * *$ \\
\hline & {$[0.02]$} & & [0.008] & \\
\hline $\begin{array}{l}\text { Adultos que habitan } \\
\text { en el hogar }\end{array}$ & -0.09 & $* *$ & -0.03 & $* *$ \\
\hline & {$[0.04]$} & & {$[0.01]$} & \\
\hline Sur-sureste & $\begin{array}{r}0.32 \\
{[0.11]}\end{array}$ & $* * *$ & $\begin{array}{r}0.12 \\
{[0.04]}\end{array}$ & $* * *$ \\
\hline Centro & $\begin{array}{r}-0.16 \\
{[0.11]}\end{array}$ & & $\begin{array}{r}-0.06 \\
{[0.04]}\end{array}$ & \\
\hline Centro-Occidente & $\begin{array}{r}-0.32 \\
{[0.11]}\end{array}$ & $* * *$ & $\begin{array}{r}-0.12 \\
{[0.04]}\end{array}$ & $* * *$ \\
\hline Noroeste & $\begin{array}{r}-0.66 \\
{[0.12]}\end{array}$ & $* * *$ & $\begin{array}{r}-0.22 \\
{[0.03]}\end{array}$ & $* * *$ \\
\hline Constante & $\begin{array}{r}0.69 \\
{[0.27]}\end{array}$ & $* * *$ & & \\
\hline Pseudo $\mathrm{R}^{2}$ & 0.12 & & & \\
\hline $\mathrm{N}$ & & & $987,994(1,745)$ & \\
\hline
\end{tabular}

$* * *, * *, *$ significativo al $1 \%, 5 \%$ y $10 \%$ respectivamente.

La estimación incorporó los factores de expansión. El número de hogares entrevistados fue de 1 745 , los cuales representan a 2987994 hogares rurales. Los errores estándar son robustos a esta corrección por factores de expansión.

Fuente: Elaboración propia. 
Resulta importante señalar que, las estimaciones presentadas en este trabajo dejan de lado cualquier efecto de equilibrio general que las remesas pudieran tener; estos efectos podrían agravar o disminuir el impacto neto de las remesas en la pobreza y la desigualdad. Queda claro que sería necesario realizar estudios más detallados para poder presentar una idea más amplia del fenómeno y del efecto neto que las remesas tienen en los ingresos de los hogares rurales.

A partir de los ejercicios realizados en este documento, los resultados muestran que las remesas provenientes de los Estados Unidos reducen la pobreza rural en México. Por otra parte es posible observar que, en cuanto a los efectos que tienen las remesas de los Estados Unidos en la distribución del ingreso, los resultados presentados indican que las remesas aumentan la inequidad en el medio rural mexicano. No obstante, el efecto es contrario para las remesas provenientes del interior del país, es decir, la emigración al resto de México reduce la inequidad.

Con base en lo anterior, es posible argumentar que la emigración rural puede promover el desarrollo rural de México. Esto sucede, sobretodo, con las remesas que reciben los hogares de los familiares que migran a los Estados Unidos y en el impacto positivo sobre la reducción de la pobreza que éstas originan.

Sin embargo, es importante señalar que la emigración hacia países desarrollados, en particular a los Estados Unidos, no debería ser la opción para mejorar las condiciones de vida de los habitantes del sector rural mexicano. Lo anterior es especialmente válido en el mundo de hoy, en el que los países receptores de migrantes internacionales están tomando medidas cada vez más drásticas para restringir el flujo de personas provenientes de los países en desarrollo. A ello hay que añadir que, una consecuencia de tales restricciones es el aumento en los riesgos que afrontan los migrantes sin permiso de entrada a los países receptores.

Por lo que, un principio básico que deben contener las políticas nacionales de desarrollo rural en México, es el de ofrecer opciones de empleo remunerativo a sus campesinos. Así como canalizar los recursos necesarios hacia la formación de capital humano, la inversión en infraestructura y comunicaciones y la promoción de actividades no agrícolas en el medio rural. 


\section{BIBLIOGRAFÍA}

CONEVAL (2006), "Nota técnica sobre la aplicación de la metodología para la medición de la pobreza por ingresos y pruebas de hipótesis 2006", <http:// www.coneval.gob.mx/contenido/med_pobreza/1017.pdf $>$.

Foster, J., J. Greer y E. Thorbecke (1984), "A Class of Decomposable Poverty Measures", Econometrica, 52(3): 761-766.

Lerman, R. y S. Yitzhaki (1985), "Income Inequality Effects by Income Source:A New Approach and Application to the U.S.", Review of Economics and Statistics, 67(1):151-56.

López Cordova E. (2005), “Globalization, Migration and Development: The role of Mexican Remittances”, Economía.

López-Feldman, A. (2006), "Decomposing inequality and obtaining marginal effects", The Stata Journal 6(1): 106-111.

Mora, J. y J. E. Taylor (2005), "Determinants of Migration, Destination and Sector Choice: Disentangling Individual, Household and Community Effects", en Çaglar Özden y Maurice Schiff (Comps.), International Migration, Remittances, and the Brain Drain, Palgrave Macmillan, NY.

Pyatt, G., C. Chen y J. Fei (1980), "The distribution of income by factor components", Quarterly Journal of Economics, 95(3): 451-73.

Stark, O., J. Edward Taylor y S. Yitzhaki (1986), "Remittances and Inequality", The Economic Journal 96:722-40.

Teruel, G., L. Rubalcava y A. Santana (2005), "Escalas de Equivalencia en México", en Números que mueven al mundo: la medición de la pobreza en México, M. Székeley (coordinador), Editorial Porrúa. 